\title{
Does the Access Angle Change the Risk of Approach- Related Complications in Minimally Invasive Lateral Lumbar Interbody Fusion? An MRI Study
}

\author{
Chunneng Huang, M.D., Zhengkuan Xu, M.D., Fangcai Li, Ph.D., Qixin Chen, Ph.D. \\ Department of Orthopaedic Surgery, The Second Affiliated Hospital of Zhejiang University School of Medicine, Hangzhou, China
}

Objective : To investigate the potential risk of approach-related complications at different access angles in minimally invasive lateral lumbar interbody fusion.

Methods : Eighty-six axial magnetic resonance images were obtained to analyze the risk of approach-related complications. The access corridor were simulated at different access angles and the potential risk of neurovascular structure injury was evaluated when the access corridor touching or overlapping the corresponding structures at each angle. Furthermore, the safe corridor length was measured when the corridor width was 18 and $22 \mathrm{~mm}$.

Results : When access angle was $0^{\circ}$, the potential risk of ipsilateral nerve roots injury was $54.7 \%$ at L4-L5. When access angle was $45^{\circ}$, the potential risk of abdominal aorta, contralateral nerve roots or central canal injury at L4-L5 was $79.1 \%, 74.4 \%$, and $30.2 \%$, respectively. The length of the $18 \mathrm{~mm}$-wide access corridor was largest at $0^{\circ}$ and it could reach $44.5 \mathrm{~mm}$ at $\mathrm{L} 3-\mathrm{L} 4$ and $46.4 \mathrm{~mm}$ at L4-L5. While the length of the $22 \mathrm{~mm}$-wide access corridor was $42.3 \mathrm{~mm}$ at L3-L4 and $44.1 \mathrm{~mm}$ at L4-L5 at $0^{\circ}$.

Conclusion : Changes in the access angle would not only affect the ipsilateral neurovascular structures, but also might adversely influence the contralateral neural elements. It should be also noted to surgeons that alteration of the access angle changed the corridor length.

Key Words : Minimally invasive lateral lumbar interbody fusion · Access angle · Access corridor · Approach-related complications . Magnetic resonance images.

\section{INTRODUCTION}

Minimally invasive lateral lumbar interbody fusion has been used increasingly as an alternative fusion method of addressing lumbar degenerative disc disease as well as spinal deformity. This approach allows to achieve the lumbar fusion with less tissue disruption by using a minimal incision. Therefore, it can have the advantages of decreased postoperative pain, minimal surgical trauma, less blood loss and faster return to daily activities ${ }^{19,21,23)}$. It can also provide better improvements in functional scores and relatively high fusion rate in treating degenerative lumbar disease ${ }^{15,19)}$. However, the approach-related complications were still bothering surgeons. Intraoperative neural and vascular structures injury was associated with regional anatom-

- Received : November 28, 2017 •Revised : December 15, 2017 •Accepted : December 17, 2017

- Address for reprints : Qixin Chen, Ph.D.

Department of Orthopaedic Surgery, The Second Affiliated Hospital of Zhejiang University School of Medicine, 88 Jiefang Road, Hangzhou 310009, China Tel : +86-187-6811-5463, Fax : +86-571-8778-3543, E-mail : zrcqx@zju.edu.cn

This is an Open Access article distributed under the terms of the Creative Commons Attribution Non-Commercial License (http://creativecommons.org/licenses/by-nc/4.0) which permits unrestricted non-commercial use, distribution, and reproduction in any medium, provided the original work is properly cited. 
ic features and might result in quadriceps muscle weakness, as well as groin and thigh numbness, even massive intraoperative bleeding $^{2,4,5,10)}$. The safety and feasibility of this approach was becoming the focus.

In order to minimize these complications, several studies have identified the positions of the nerve roots and retroperitoneal great vessels in relation to the intervertebral disc at each level. The concept of "safe zone" was introduced to describe the access corridor as well as the distance between ventral nerves and retroperitoneal vessels. The width of the safe zone was thought to be narrowed at L4-L5 due to the more anteriorly located nerve root when it goes down the body ${ }^{14,26)}$. However, these studies only focused on the access corridor on the direct lateral orientation. The corridor length was still unknown when the access angle changed. As oblique lumbar interbody fusion was gaining its popularity these years, the selection of the access angle became diverse. However, the transverse section of intervertebral disc was an irregular oval instead of circle shape. The corridor length might be variable at different access angles. An inappropriate access angle or corridor length might induce neural or vascular complications. Previous studies have reported the ipsilateral and contralateral nerve injury in minimally invasive lateral approach due to the inappropriate access corridor as well as misaligned cage ${ }^{1,7,22,25)}$. Papanastassiou et al. ${ }^{22)}$ suggested the occurrence of these injuries might be associated with the access angle. Therefore, it is imperative to analyze the impact of the access angle on the incidence of neurovascular structure injury and measure the corridor length at each access angle. But as far as we know, no available anatomic researches have analyzed it.

Therefore, we had two objectives in this study. The primary goal of this study was to investigate the potential risk of ipsilateral or contralateral neurovascular structure injury at different access angles including abdominal aorta, nerve roots, genitofemoral nerve as well as central canal. The secondary objective was to define the corridor length at different access angles in minimally invasive lateral lumbar interbody fusion.

\section{MATERIALS AND METHODS}

\section{Inclusion and exclusion criteria}

We enrolled the patients who had ever lumbar spine examination using magnetic resonance imaging (MRI) due to radicular pain, low back pain or intermittent claudication. The pa- tients who had the history of spine surgery, spinal deformity, spondylolisthesis, trauma, tumor or lumbar infection were excluded from this research. Furthermore, the patients in whom the distance between left nerve roots and retroperitoneal great vessels was smaller than $18 \mathrm{~mm}$ were also excluded as they were considered to be unsuitable for this approach. From February 2013 to May 2017, 86 patients consisting of 49 males and 37 females were retrospectively reviewed in present study with an average age of $52.6 \pm 11.6$ years old. This research was permitted by Ethnics Committee (No. 2017-047).

\section{Image analysis}

A 1.5-Tesla lumbar spine magnetic resonance scan (Siemens Corporation, Munich, Germany) was performed from L2-L3 to L4-L5 level in these patients. Sagittal T1-/T2- and T2 weighted fat suppressed sequences were obtained at 3.0-3.5 mm slice intervals without a gap. Axial T2 weighted images were also obtained continuously through spine, rightly angled to the disc space with 2.5-3.0 mm slice intervals. The left approach was preferred by surgeons in lateral approach, as the working zone was larger in this side ${ }^{14,26)}$. In addition, the lumbar vertebrae of L3-L4 and L4-L5 were likely to develop symptomatic degenerative process in these two levels ${ }^{6,8)}$. Therefore, we only focused on L3-L4 and L4-L5 levels from the left side. Morphological parameters were measured from axial T2 weighted images at mid-disc level cuts of L3-L4 and L4-L5 using Image J software (version 1.4; National Institutes of Health, Bethesda, MD, USA).

\section{The potential risk of the neurovascular structures injury at different access angles}

The disc center was defined as the intersection of the coronal central perpendicular line and sagittal central perpendicular line (SCPL) in the cross section of the disc. Based on the disc center, the rectangle was delineated to simulate the access corridor, the long dimension of which was parallel to the SCPL. The width of the rectangle was $18 \mathrm{~mm}$ while the length of it was limited to avoid exceeding the disc border. The left extension lines were also depicted to simulate the working channel. Then, this rectangle along with the extension lines rotated around the disc center at an angle of $0^{\circ}, 5^{\circ}, 10^{\circ}, 15^{\circ}$, $30^{\circ}$, and $45^{\circ}$ without changing the length or width. The access angle was defined as the angle of the long dimension of the rectangle to SCPL (Fig. 1A). We intended to observe and re- 
cord the potential risk of nerve roots, abdominal aorta, central canal or genitofemoral nerve injury, which was defined as the rectangle or the space between extension lines touching or overlapping the corresponding structures.

\section{The position of the access axis relative to genito- femoral nerve and potential risk of genitofemoral nerve injury at different access angles}

The area between anterior edge of the disc and the SCPL was divided into four zones on average. The most anterior zone was defined as zone I, the second anterior zone was zone II, the third anterior area was zone III, and the most posterior area was zone IV. A rectangle was delineated to act as the access corridor, the long dimension of which was parallel to SCPL. The width of the rectangle was $18 \mathrm{~mm}$ while the length of it was limited to avoid exceeding the disc border. Then, this rectangle rotated around the disc center and the length of it was adjusted to avoid exceeding the disc border. The axis of the rectangle was also depicted as the access axis. The access point was defined as the intersection of access axis and the disc border (Fig. 1B). The position of the access axis relative to genitofemoral nerve was recorded at an angle of $0^{\circ}, 5^{\circ}, 10^{\circ}, 15^{\circ}, 30^{\circ}$, and $45^{\circ}$. In addition, the distribution of the access points was analyzed at each zone.

\section{Measurements of the length of the rectangle with different widths at different access angles}

A rectangle was delineated to act as the access corridor, the long dimension of which was parallel to SCPL. The width of the rectangle was $18 \mathrm{~mm}$ while the length of it was limited to avoid exceeding the disc border. Then, this rectangle rotated around the disc center at an angle of $0^{\circ}, 5^{\circ}, 10^{\circ}, 15^{\circ}, 30^{\circ}$, and $45^{\circ}$. Meanwhile, the length of this rectangle was also adjusted to avoid exceeding the border of the disc and it was measured at different access angles. Similar measurements were also performed for the length when the width of the rectangle extended from 18 to $22 \mathrm{~mm}$ (Fig. 2).

\section{Statistical analysis}

SPSS version 22 (IBM, Chicago, IL, USA) was employed to perform statistical analysis. Fisher exact test was performed to compare the difference of the potential risk of the neurovascular structure injury among access angles. An unpaired student $\mathrm{t}$
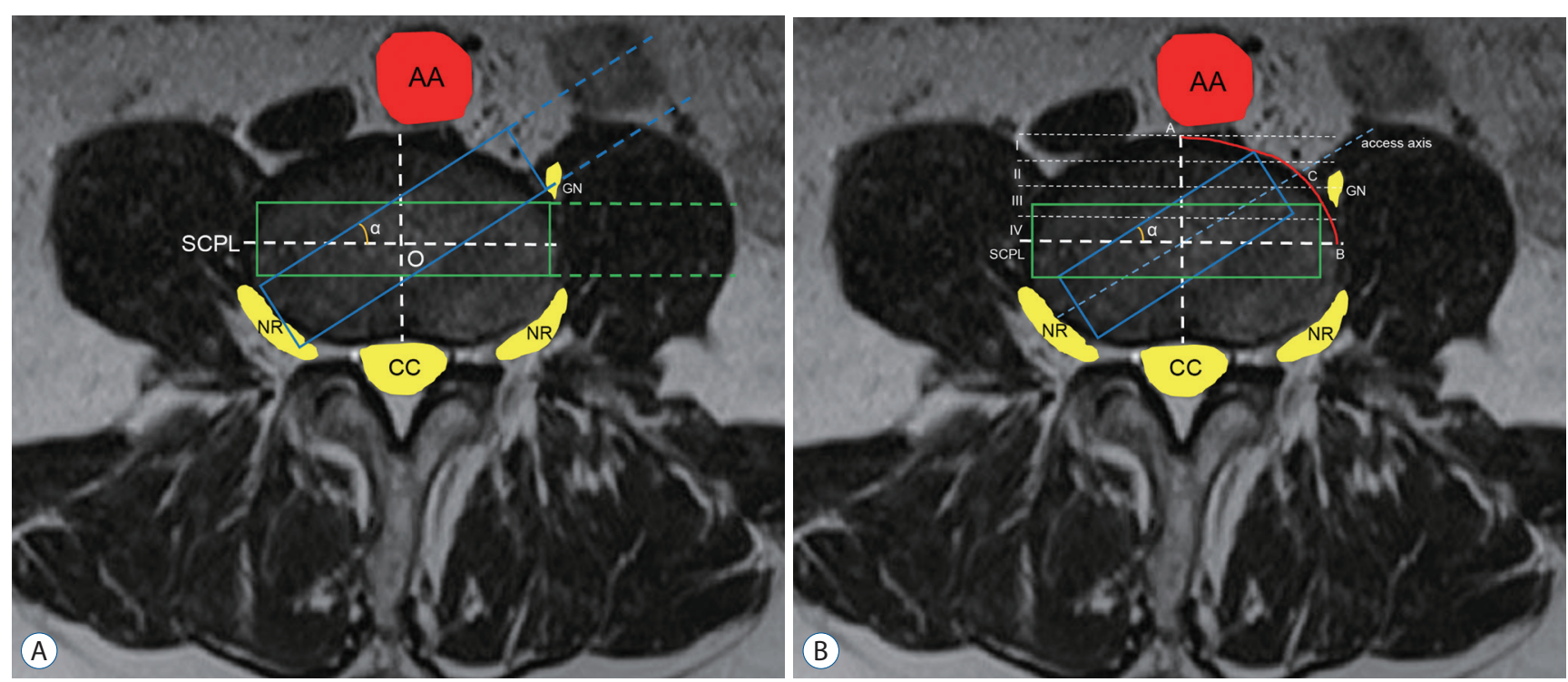

Fig. 1. The potential risk of the neurovascular structures injury at different access angles at $L 3-L 4$ level. A: The long dimension of the green rectangle with $18 \mathrm{~mm}$ width was parallel to SCPL. The green dotted lines indicated the left extension lines of the green rectangle. The blue rectangle along with the blue dotted lines came from the green rectangle rotating around the disc center with angle $a$. The potential risk of neurovascular injury was recorded at the angle a of $0^{\circ}, 5^{\circ}, 15^{\circ}, 30^{\circ}$ and $45^{\circ}$ if the rectangle and the space between extension line touched or overlapped these structures. $B$ : The area between anterior edge of the disc and the SCPL was divided into four zones. The long dimension of the green rectangle was parallel to SCLP. The blue rectangle came from the green rectangle rotating around the disc center with angle $a$ and the length of it was adjusted to avoid exceeding the border of the disc. The blue dotted line was the axis of the blue rectangle and it intersected the disc border (AB) at the access point (C). The distribution of the access point at each zone and the relative position between access axis and the genitofemoral nerve were analyzed. AA : abdominal aorta, GN : genitofemoral nerve, SCPL : sagittal central perpendicular line, $\mathrm{O}$ : disc center, NR: nerve roots, CC: central canal. 
test was used to compare the differences of the length of the rectangle between levels. One-way analysis of variance with a post hoc Tukey test was used to analyze the length of the rectangle among different access angles. $p<0.05$ was considered significant.

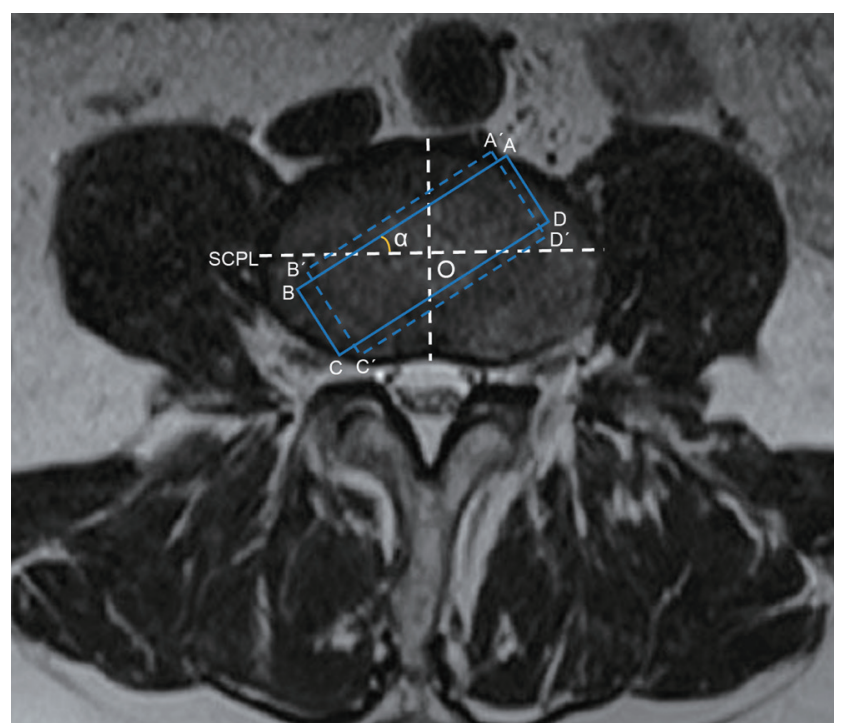

Fig. 2. Measurements of the corridor length at different access angles and widths. $A B C D$ came from the rectangle rotating around the disc center with an angle $a$, long dimension of which was parallel to SCPL. $A^{\prime} B^{\prime} C^{\prime} D^{\prime}$ indicated the rectangle with the width extending from $18 \mathrm{~mm}$ to $22 \mathrm{~mm}$. The length of the rectangle with $18 \mathrm{~mm}$ width $(A B)$ and $22 \mathrm{~mm}$ width $\left(A^{\prime} B^{\prime}\right)$ was measured at the angle $a$ of $0^{\circ}, 5^{\circ}, 15^{\circ}, 30^{\circ}$, and $45^{\circ}$. SCPL : sagittal central perpendicular line, $\mathrm{O}$ : disc center.

\section{RESULTS}

\section{Potential risk of the neurovascular structures injury at different access angles}

The ipsilateral nerve roots might get injured when the access angle was $0^{\circ}$, especially at L4-L5 (54.7\%). When the access angle was $5^{\circ}$, the potential risk of ipsilateral nerve roots injury decreased (3.5\% at L3-L4, 8.1\% at L4-L5), but the abdominal aorta might be influenced at L4-L5 (1.2\%). When the access angle increased to $15^{\circ}$, the potential risk of contralateral nerve roots became higher $(12.8 \%$ at $\mathrm{L} 3-\mathrm{L} 4,17.4 \%$ at L4-L5). The central canal would get injured only at $45^{\circ}$ in both levels $(40.7 \%$ at $\mathrm{L} 3-\mathrm{L} 4,30.2 \%$ at $\mathrm{L} 4-\mathrm{L} 5)$ and the potential risk of contralateral nerve roots injury reached its maximum $(61.6 \%$ at L3-L4, $74.7 \%$ at L4-L5) (Table 1).

\section{The position of the access axis relative to genito- femoral nerve and potential risk of genitofemoral nerve injury at different access angles}

When the access angle was $0^{\circ}$, all the access points located at zone IV and most of the access axis was posterior to genitofemoral nerve (93.0\% at L3-L4, 100.0\% at L4-L5). The genitofemoral nerve might be influenced by the working channel, especially at L3-L4 (61.6\%). When the access angle increased to $15^{\circ}$, the access points might locate at zone III and $51.2 \%$ access axis would go across the genitofemoral nerve at L3-L4. The potential risk of genitofemoral nerve injury was extremely high at both levels (100.0\% at L3-L4, 96.5\% at L4-L5). When the

Table 1. The potential risk of the neurovascular structures injury at different access angles

\begin{tabular}{|c|c|c|c|c|c|c|c|}
\hline & $0^{\circ}$ & $5^{\circ}$ & $10^{\circ}$ & $15^{\circ}$ & $30^{\circ}$ & $45^{\circ}$ & $p$-value* \\
\hline \multicolumn{8}{|l|}{ L3-L4 } \\
\hline Ipisilateral nerve root injury & 16/86 (18.6) & $3 / 86(3.5)$ & $0 / 86(0.0)$ & $0 / 86(0.0)$ & $0 / 86(0.0)$ & $0 / 86(0.0)$ & $<0.001$ \\
\hline Abdominal aorta injury & $0 / 86(0.0)$ & 0/86 (0.0) & $0 / 86(0.0)$ & $0 / 86(0.0)$ & $4 / 86(4.7)$ & $56 / 86(65.1)$ & $<0.001$ \\
\hline Contralateral nerve root injury & $0 / 86(0.0)$ & $1 / 86(1.2)$ & $6 / 86(7.0)$ & $11 / 86(12.8)$ & $37 / 86(43.0)$ & 53/86 (61.6) & $<0.001$ \\
\hline Central canal injury & $0 / 86(0.0)$ & $0 / 86(0.0)$ & $0 / 86(0.0)$ & $0 / 86(0.0)$ & $0 / 86(0.0)$ & $35 / 86(40.7)$ & $<0.001$ \\
\hline \multicolumn{8}{|l|}{$\llcorner 4-\llcorner 5$} \\
\hline Ipisilateral nerve root injury & $47 / 86(54.7)$ & $7 / 86(8.1)$ & $0 / 86(0.0)$ & $0 / 86(0.0)$ & $0 / 86(0.0)$ & $0 / 86(0.0)$ & $<0.001$ \\
\hline Abdominal aorta injury & $0 / 86(0.0)$ & $1 / 86(1.2)$ & $6 / 86(7.0)$ & $13 / 86(15.0)$ & $43 / 86(50.0)$ & $68 / 86(79.1)$ & $<0.001$ \\
\hline Contralateral nerve root injury & $0 / 86(0.0)$ & 2/86 (2.3) & $8 / 86(9.3)$ & 15/86 (17.4) & $45 / 86(52.3)$ & $64 / 86(74.4)$ & $<0.001$ \\
\hline Central canal injury & $0 / 86(0.0)$ & 0/86 (0.0) & $0 / 86(0.0)$ & $0 / 86(0.0)$ & $0 / 86(0.0)$ & $26 / 86(30.2)$ & $<0.001$ \\
\hline
\end{tabular}

Values are presented as $\mathrm{n} / \mathrm{N}$ (\%). The neurovascular structures injury was determined by the rectangle and the space between extension lines touching or overlapping these structures at different access angles according to Fig. 1A. *Comparison for the potential risk of injuring to each structure among different access angles using Fisher exact test 
access angle was $30^{\circ}$, the access axis still had high possibility of pricking on genitofemoral nerve at L4-L5 (50.0\%) and all the access points located at zone II. When the access angle was $45^{\circ}$, the potential risk of genitofemoral nerve injury decreased $(3.5 \%$ at L3-L4, 20.9\% at L4-L5) (Fig. 3).
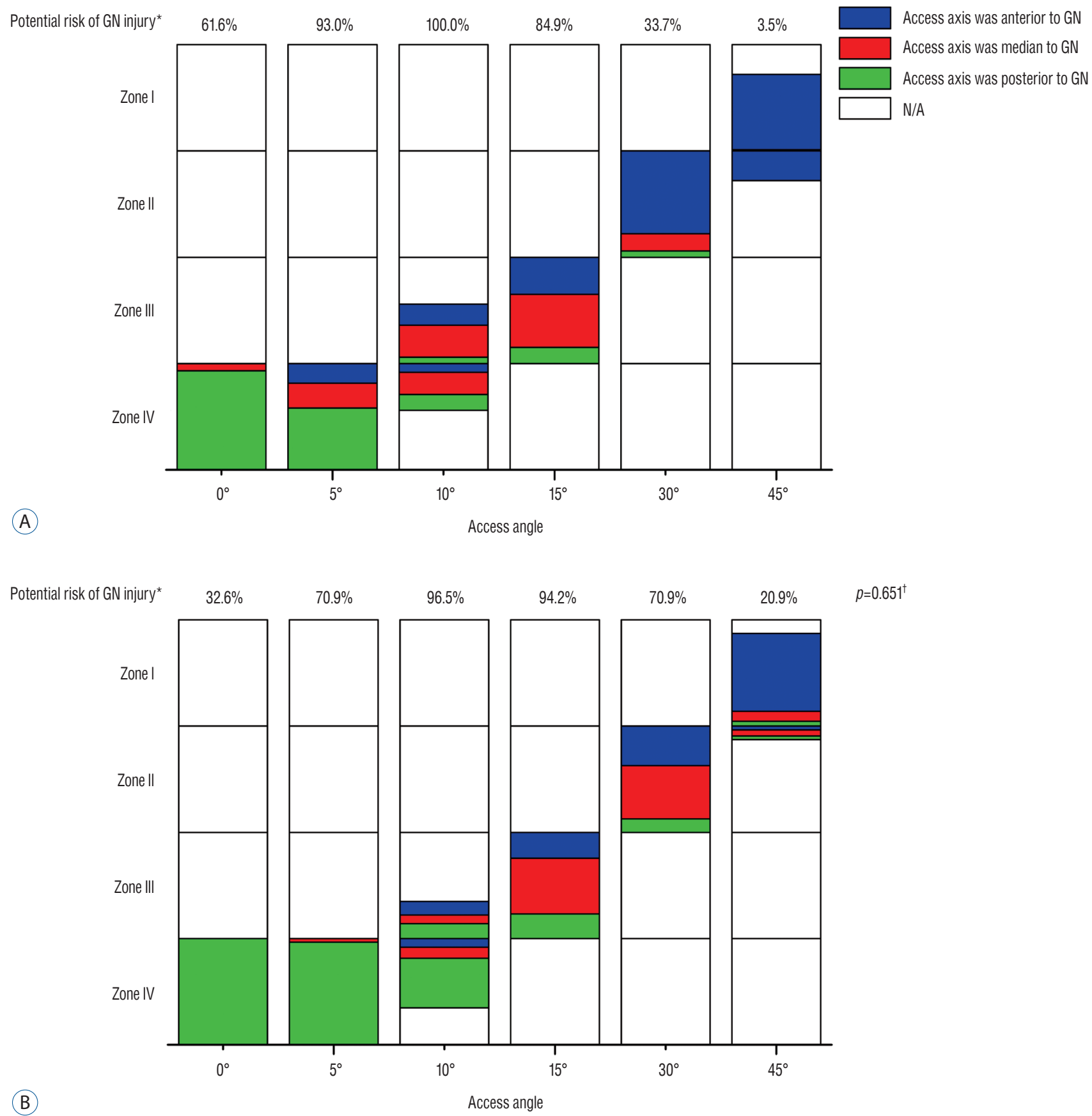

Fig. 3. The position of the access axis relative to genitofemoral nerve and potential risk of genitofemoral nerve injury at different access angles. (A) and (B) indicated the measurements of relevant parameters at $L 3-L 4$ and $L 4-L 5$, respectively. The blue, red or green color indicated the access axis was anterior, median or posterior to genitofemoral nerve, respectively. The location of these colors indicated access points at each zone. *The comparison for the potential risk of genitofemoral nerve injury between $\mathrm{L} 3-\mathrm{L} 4$ and $\mathrm{L} 4-\mathrm{L} 5$ at all access angles using Fisher exact test. ${ }^{\dagger} T$ The genitofemoral nerve injury was determined by the rectangle and the space between extension line touching or overlapping it at different access angles according to Fig. 1A. GN: genitofemoral nerve, N/A : not available. 
Table 2. The length of the rectangle with different widths at different access angles

\begin{tabular}{ccccccccc}
\hline & Width $(\mathbf{m m})$ & $\mathbf{0}^{\circ}$ & $\mathbf{5}^{\circ}$ & $\mathbf{1 0}^{\circ}$ & $\mathbf{1 5}^{\circ}$ & $\mathbf{3 0}^{\circ}$ & $\mathbf{4 5}^{\circ}$ & $\boldsymbol{p}_{\text {-value }}$ \\
\hline L3-L4 & 18 & $44.5 \pm 3.8$ & $43.7 \pm 3.6$ & $42.9 \pm 3.4$ & $42.0 \pm 3.5$ & $38.8 \pm 3.2$ & $34.5 \pm 3.3$ & $<0.001^{*}$ \\
& 22 & $42.3 \pm 3.9$ & $41.7 \pm 3.7$ & $40.7 \pm 3.4$ & $39.7 \pm 3.4$ & $37.3 \pm 3.2$ & $32.3 \pm 2.9$ & $<0.001^{\dagger}$ \\
& $p$-value & $<0.001$ & $<0.001$ & $<0.001$ & $<0.001$ & 0.001 & $<0.001$ & $36.2 \pm 3.5$ \\
L4-L5 & 18 & $46.4 \pm 3.8$ & $45.9 \pm 3.7$ & $45.1 \pm 3.7$ & $43.7 \pm 3.7$ & $40.6 \pm 3.4$ & $<0.001^{\dagger}$ \\
& 22 & $44.1 \pm 3.8$ & $43.4 \pm 3.7$ & $42.6 \pm 3.6$ & $41.3 \pm 3.7$ & $38.3 \pm 3.2$ & $34.1 \pm 3.1$ & $<0.001^{\dagger}$ \\
& $p$-value & $<0.001$ & $<0.001$ & $<0.001$ & $<0.001$ & $<0.001$ & $<0.001$ & \\
\hline
\end{tabular}

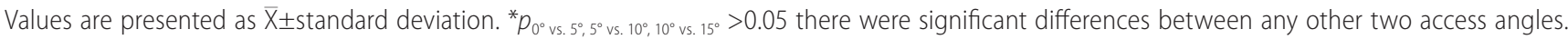

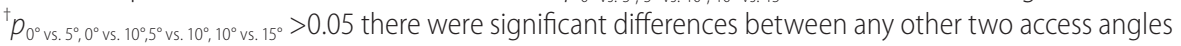

\section{The length of the rectangle with different widths at different access angles}

The length of the rectangle at different access angles was showed in Table 2. For L3-L4, the length of the rectangle with $18 \mathrm{~mm}$ width could reach $40 \mathrm{~mm}$ when the access angle was $15^{\circ}(42.0 \pm 3.5 \mathrm{~mm})$. But the access angle should be smaller than $10^{\circ}$ to acquire $40 \mathrm{~mm}$ length with $22 \mathrm{~mm}$ width $(40.7 \pm 3.4 \mathrm{~mm})$. The length could be close to $45 \mathrm{~mm}$ when the width of the rectangle was $18 \mathrm{~mm}$ and the access angle was $0^{\circ}(44.5 \pm 3.8 \mathrm{~mm})$.

In order to acquire a $40 \mathrm{~mm}$ length at L4-L5, the access angle should be smaller $30^{\circ}$ with an $18 \mathrm{~mm}$ width $(40.6 \pm 3.4 \mathrm{~mm})$ and it should be no more than $15^{\circ}$ with a $22 \mathrm{~mm}$ width (41.3 \pm $3.7 \mathrm{~mm}$ ). When the access angle was smaller than $10^{\circ}$, the length of the rectangle with $18 \mathrm{~mm}$ width could be larger than $45 \mathrm{~mm}$ at L4-L5 $(45.1 \pm 3.7 \mathrm{~mm})$. As for $22 \mathrm{~mm}$ wide rectangle, the length would be close to $45 \mathrm{~mm}$ when the access angle was $0^{\circ}(44.1 \pm 3.8 \mathrm{~mm})$ and any access angle alteration would decrease the length. Generally, L4-L5 provided larger length than that at $\mathrm{L} 3-\mathrm{L} 4$.

\section{DISCUSSION}

Although minimally invasive lateral lumbar interbody fusion had the advantages of less muscular or ligamentous dissection, shorter hospital stay and faster recovery with good clinical outcomes ${ }^{19,21)}$, the high incidence of ipsilateral or contralateral nerve damage as well as major vascular complications were still reported by a number of clinical researches ${ }^{3,13,23,25)}$. In order to reduce these complications, several anatomical researches have worked on the access corridor in relation to lateral approach ${ }^{11,12,26)}$. However, they only analyzed the access corridor at $0^{\circ}$ access angle. To the best of our knowledge, there were still no published MRI studies that described the access corridor at different access angles and fully revealed the relationship between different access angles and approach-related complications. In this study, we simulated the access corridor at different access angles and evaluated the relative position between the access corridor and the ipsilateral or contralateral neurovascular structures. Then, the potential risk of neurovascular structures injury at different access angles was analyzed and we found the following features : 1) the access corridor might not only affect the ipsilateral nerves and vessels, but also would adversely influence the contralateral nerve roots or even central canal when the access angle changed. 2) The potential risk of the nerve roots or abdominal aorta injury varied at different access angles. The potential risk of ipsilateral nerve root injury was high at a small access angle, while the abdominal aorta as well as the contralateral nerve root might be easily influenced at a large angle. Therefore, it is necessary to choose an appropriate access angle to reduce the perioperative complications.

Direct injury to the ipsilateral nerve root and great vessels is the most concerning complication in lateral lumbar interbody fusion. Previous anatomical study demonstrated that the distance between abdominal aorta and left nerve roots was 28.57 $\mathrm{mm}$ at $\mathrm{L} 3-\mathrm{L} 4$ and $22.63 \mathrm{~mm}$ at L4-L5 ${ }^{26}$, which seemed to be large enough for the establishment of a $22 \mathrm{~mm}$-wide corridor. Based on our measurements, the $18 \mathrm{~mm}$-wide corridor was feasible only when the access angles was between $5^{\circ}$ and $15^{\circ}$. With a $5^{\circ}$ or smaller angle, the potential risk of ipsilateral nerve root injury at L3-L4 and L4-L5 was up to $18.6 \%$ and 54.7\%, respectively. On the other hand, the abdominal aorta would be easily influenced with the angle larger than $15^{\circ}$. These results indicated the access angle was a crucial factor for the occurrence of the ipsilateral neurovascular structure injury. Besides, 
this could also explain the fact that the direct lateral approach had higher risk of nerve roots injury while more major vessels injuries were observed in the oblique approach ${ }^{10)}$. Therefore, in addition to the corridor width, surgeons should pay more attention to the selection of appropriate access angle to reduce the approach-related complications. The present study suggested the access angle between $5^{\circ}$ and $15^{\circ}$ was safe for both ipsilateral nerve roots and abdominal aorta. When using an access angle smaller than $5^{\circ}$, direct penetration to the ipsilateral nerve roots should be avoided. While establishing the access corridor with an access angle larger than $15^{\circ}$, the protection of the major vessels was the priority.

Another documented risk in minimally invasive lateral approach was damage to the genitofemoral nerve ${ }^{20)}$. A precise knowledge of the position of the genitofemoral nerve and performing an appropriate retraction might be critical to reduce this complication ${ }^{6}$. However, the image analysis in the present study showed the position of the genitofemoral nerve was various among patients. In particular, the access axis had high possibility of pricking on it when the access angle was between $10^{\circ}$ and $30^{\circ}$. This result was supported by the previous results presented in cadaveric and clinical studies that the genitofemoral nerve palsy was likely to take place even though the imaging anatomy of it was evaluated before surgery ${ }^{4,6,18)}$. Because the genitofemoral nerve pierced psoas muscles and emerged from anterior surface of psoas as it went down the body ${ }^{6}$, direct visualization and soft retraction nerve retraction technique of the genitofemoral nerve might be a better option to reduce this complication ${ }^{6}$. He et al. ${ }^{12)}$ analyzed the course of the genitofemoral nerve and suggested that a posterior retraction of the genitofemoral nerve was required as it enabled neural tissues to remain relaxed. However, according to our results, the access axis was generally located posterior to the genitofemoral nerve at the angle smaller than $10^{\circ}$. In this condition, if the access corridor was established anteriorly to the genitofemoral nerve just as He et al. ${ }^{12}$ suggested, there would be high possibilities of direct neural retraction. What's more, the retraction distance of the genitofemoral nerve would be also increased, which would contribute to the occurrence of genitofemoral nerve injury. Therefore, we suggested that retractions of the genitofemoral nerve should be based on the access angle.

In order to acquire larger surface area for graft retention and decrease the risk of subsidence, appropriate access corridor that allowed for implanting a long and wide cage to span across both bilateral margins of the apophyseal ring was critical to perform a successful lateral interbody fusion ${ }^{16,17)}$. Nevertheless, an excessively long corridor would exceed the disc border and might cause damage to the contralateral nerve root or even central canal ${ }^{9,22,24,25)}$. In present study, the potential risk of contralateral nerve root injury at L4-L5 at $15^{\circ}, 30^{\circ}$, and $45^{\circ}$ was $17.4 \%, 52.3 \%$, and $74.4 \%$, respectively. Moreover, the potential risk of injury to central canal was as high as $30.2 \%$ at $45^{\circ}$. Because the transverse section of the disc was an oval, the access corridor might protrude beyond the disc space if the corridor length and width remained constant at different access angles. This signified that the access angle might be an important factor to cause contralateral nerve injuries. For the sake of reducing this complication, the corridor length or corridor width should be decreased when the access angle got larger. Besides, our study also showed that the safe corridor length with an 18 mm width was significantly larger than that with a $22 \mathrm{~mm}$ width at all angles. Therefore, it was reasonable to reduce the width of the cage in order to acquire a long enough cage.

Of course, there are a number of limitations to this study. First, this was a magnetic resonance imaging-based study in which the procedure was performed in supine position, but lateral lumbar interbody fusion was performed in lateral decubitus position. Therefore, measurements of relevant parameters in present study may have deviations. However, this deviation will not dramatically affect the trends of the potential risk of neurovascular injury at each angle. Second, measurements in this study only involved L3-L4 and L4-L5 levels. Although these two levels are commonly involved in degenerative spinal disorders, similar evaluation at L1-L2 as well as L2-L3 would be useful. Third, the image analysis might be different from the actual surgical conditions. Because of the use of the retraction, some structures such as genitofemoral nerve and abdominal aorta could be mobilized. Nevertheless, this is the first study to describe the relative position between the access corridor and the neurovascular structures at different access angle, and the anatomic information may be still useful to conduct the application of retractions prior to lateral lumbar interbody fusion procedure and help reduce injury to the neurovascular structures.

In conclusion, changes the access angle would not only affect the ipsilateral neurovascular structures, but also might adversely influence the contralateral neural elements. Meanwhile, the safe corridor length was also different when the access angle 
changed. An excessively small access angle increased the risk of ipsilateral nerve root injury, while a large angle posed high risk to abdominal aorta as well as the contralateral nerve roots. We recommended that the access angle between $5^{\circ}$ and $15^{\circ}$ was safe for lateral lumbar interbody fusion. Direct penetration to the ipsilateral nerve roots should be avoided with the access angle smaller than $5^{\circ}$, while the protection of major vessels was the priority with the access angle larger than $15^{\circ}$.

\section{CONCLUSION}

GKR could be an additional treatment option in recurrent high-grade glioma, particularly in patients with good PS and small tumor volume.

\section{CONFLICTS OF INTEREST}

No potential conflict of interest relevant to this article was reported.

\section{INFORMED CONSENT}

Informed consent was obtained from all individual participants included in this study.

\section{- Acknowledgements}

This study was supported by the National Natural Science Foundation of China (grant No. 81472114).

\section{References}

1. Abe K, Orita S, Mannoji C, Motegi H, Aramomi M, Ishikawa T, et al. : Perioperative complications in 155 patients who underwent oblique lateral interbody fusion surgery: perspectives and indications from a retrospective, multicenter survey. Spine (Phila Pa 1976) 42 : 55-62, 2017

2. Ahmadian A, Deukmedjian AR, Abel N, Dakwar E, Uribe JS : Analysis of lumbar plexopathies and nerve injury after lateral retroperitoneal transpsoas approach: diagnostic standardization. J Neurosurg Spine 18 : 289-297, 2013

3. Aichmair A, Fantini GA, Garvin S, Beckman J, Girardi FP : Aortic perforation during lateral lumbar interbody fusion. J Spinal Disord Tech 28 :
71-75, 2015

4. Anand N, Rosemann R, Khalsa B, Baron EM : Mid-term to long-term clinical and functional outcomes of minimally invasive correction and fusion for adults with scoliosis. Neurosurg Focus 28 : E6, 2010

5. Assina R, Majmundar NJ, Herschman Y, Heary RF : First report of major vascular injury due to lateral transpsoas approach leading to fatality. J Neurosurg Spine 21 : 794-798, 2014

6. Banagan K, Gelb D, Poelstra K, Ludwig S : Anatomic mapping of lumbar nerve roots during a direct lateral transpsoas approach to the spine: a cadaveric study. Spine (Phila Pa 1976) 36 : E687-E691, 2011

7. Beckman JM, Vincent B, Park MS, Billys JB, Isaacs RE, Pimenta L, et al. : Contralateral psoas hematoma after minimally invasive, lateral retroperitoneal transpsoas lumbar interbody fusion: a multicenter review of 3950 lumbar levels. J Neurosurg Spine 26 : 50-54, 2017

8. Berjano P, Balsano M, Buric J, Petruzzi M, Lamartina C : Direct lateral access lumbar and thoracolumbar fusion: preliminary results. Eur Spine J 21 Suppl 1 : S37-S42, 2012

9. Chang J, Kim JS, Jo H : Ventral dural injury after oblique lumbar interbody fusion. World Neurosurg 98 : 881.e1-881.e4, 2017

10. Fujibayashi S, Kawakami N, Asazuma T, Ito M, Mizutani J, Nagashima H, et al. : Complications associated with lateral interbody fusion: nationwide survey of 2998 cases during the first two years of its use in Japan. Spine (Phila Pa 1976) 42 : 1478-1484, 2017

11. Guérin P, Obeid I, Gille O, Bourghli A, Luc S, Pointillart V, et al. : Safe working zones using the minimally invasive lateral retroperitoneal transpsoas approach: a morphometric study. Surg Radiol Anat 33 : 665671, 2011

12. He L, Kang Z, Tang WJ, Rong LM : A MRI study of lumbar plexus with respect to the lateral transpsoas approach to the lumbar spine. Eur Spine J 24 : 2538-2545, 2015

13. Hijji FY, Narain AS, Bohl DD, Ahn J, Long WW, DiBattista JV, et al. : Lateral lumbar interbody fusion: a systematic review of complication rates. Spine J 17 : 1412-1419, 2017

14. Kepler CK, Bogner EA, Herzog RJ, Huang RC : Anatomy of the psoas muscle and lumbar plexus with respect to the surgical approach for lateral transpsoas interbody fusion. Eur Spine J 20 : 550-556, 2011

15. Kim JS, Choi WS, Sung JH : 314 minimally invasive oblique lateral interbody fusion for L4-5: clinical outcomes and perioperative complications. Neurosurgery 63 Suppl 1 : 190-191, 2016

16. Le TV, Baaj AA, Dakwar E, Burkett CJ, Murray G, Smith DA, et al. : Subsidence of polyetheretherketone intervertebral cages in minimally invasive lateral retroperitoneal transpsoas lumbar interbody fusion. Spine (Phila Pa 1976) 37 : 1268-1273, 2012

17. Marchi L, Abdala N, Oliveira L, Amaral R, Coutinho E, Pimenta L : Radiographic and clinical evaluation of cage subsidence after stand-alone lateral interbody fusion. J Neurosurg Spine 19 : 110-118, 2013

18. Moller DJ, Slimack NP, Acosta FL Jr, Koski TR, Fessler RG, Liu JC : Minimally invasive lateral lumbar interbody fusion and transpsoas approachrelated morbidity. Neurosurg Focus 31 : E4, 2011

19. Molloy S, Butler JS, Benton A, Malhotra K, Selvadurai S, Agu 0 : A new extensile anterolateral retroperitoneal approach for lumbar interbody fu- 
sion from L1 to S1: a prospective series with clinical outcomes. Spine J 16 : 786-791, 2016

20. O'Brien J, Haines C, Dooley ZA, Turner AW, Jackson D : Femoral nerve strain at L4-L5 is minimized by hip flexion and increased by table break when performing lateral interbody fusion. Spine (Phila Pa 1976) 39 : 33-38, 2014

21. Ozgur BM, Aryan HE, Pimenta L, Taylor WR : Extreme lateral interbody fusion (XLIF): a novel surgical technique for anterior lumbar interbody fusion. Spine J 6 : 435-443, 2006

22. Papanastassiou ID, Eleraky M, Vrionis FD : Contralateral femoral nerve compression: An unrecognized complication after extreme lateral interbody fusion (XLIF). J Clin Neurosci 18 : 149-151, 2011

23. Phan K, Maharaj M, Assem Y, Mobbs RJ : Review of early clinical results and complications associated with oblique lumbar interbody fusion (OLIF). J Clin Neurosci 31 : 23-29, 2016

24. Regev GJ, Haloman S, Chen L, Dhawan M, Lee YP, Garfin SR, et al. : Incidence and prevention of intervertebral cage overhang with minimally invasive lateral approach fusions. Spine (Phila Pa 1976) 35 : 14061411, 2010

25. Taher $F$, Hughes AP, Lebl DR, Sama AA, Pumberger $M$, Aichmair A, et al. : Contralateral motor deficits after lateral lumbar interbody fusion. Spine (Phila Pa 1976) 38 : 1959-1963, 2013

26. Yusof MI, Nadarajan E, Abdullah MS : The morphometric study of I3-L4 and L4-L5 lumbar spine in Asian population using magnetic resonance imaging: feasibility analysis for transpsoas lumbar interbody fusion. Spine (Phila Pa 1976) 39 : E811-E816, 2014 